\title{
Theory of Intermolecular Interaction
}

\author{
Shigeyuki Aono ${ }^{\#}$ \\ Division of theoretical chemistry, Faculty of Science, Kanazawa University, Kanazawa, Ishikawa, 920-1192
}

(Received June 18, 2001)

\begin{abstract}
The method of orbital interaction describing the intermolecular interaction is reformulated in terms of Green's function. The eigen-values and eigen-vectors for individual molecules obtained by block diagonalization of the original Hamiltonian, are sufficient to predict the next step of the reaction. The polarizabilities in the ordinary perturbation treatment is also effectively used. This procedure is further clarified by defining the effective coupling. The method is applied to a molecular compound between ethylene and $\mathrm{H}_{2}$.
\end{abstract}

There are various types of chemical reactivity ${ }^{1,2,3}$. If any perturbation $v$ is put on a system described by the Hamiltonian $H^{0}$, the expected reaction can be presented in terms of $H^{0}$ and $v$. In the course of theory, indices useful for indicating reactivity are offered: the charge density, bond order and polarizability in Coulson's theory, the HOMO and LUMO orbitals in Fukui's theory, and symmetry conservation in Woodward-Hoffmann's theory. In order to synthesize a new compound from $A$ and $B$, the theory of chemical reactivity turns to the theory of orbital interaction $^{2,4}$. Namely, the Hamiltonians for $A$ and $B$ are already diagonalized, and their eigen-values and eigen-functions are prepared; if some interaction between $A$ and $B$ is supposed, the problem is what reaction can be expected. The mechanism responsible to this reaction must be described by couplings between eigenfunctions of $A$ and $B$.

In this paper, Coulson's theory is reformulated in terms of Green's function. Because Coulson's theory is, itself, an old fashioned Green's function theory, it is now dressed in the latest style. We have successfully unified this theory with other theories.

In chapter 1 , we briefly sketch our general treatment of the chemical ractivity ${ }^{11,12}$. This is applied in chapter 2 to inter-molecular interaction, where the elucidated mechanism corresponds to the second-order perturbation theory of the ordinary perturbation theory, so that the polarizability in Coulson's theory takes place. In chapter 3 , an effective interaction obtained by including the molecular interaction is given and facilitates one to recognize the intermolecular interaction. In chapter 4, the problem in the radiation field is treated. Finally, some applications are briefly discussed.

\section{General}

Let us consider a problem described by the Hamiltonian $H$. It seems to be almost impossible to solve this eigenvalue problem exactly. However, if we divide $H$ into two parts,

$$
H=H^{0}+v
$$

\# Professor emeritus of Kanazawa university. Present address: Funabashi Fujiwara 7-41-2-102, Chiba 273-0047 the solution for the non-interacting part $H^{0}$ is usually given by

$$
H^{0}=\left|i>\epsilon_{\mathrm{i}}<i\right|
$$

We define the Green's operator for $H$,

$$
G(z)=\frac{1}{z-H}
$$

and for $H^{0}$

$$
G^{0}(z)=\frac{1}{z-H^{0}}
$$

The total energy for $H^{0}$ is expressed by

$$
\begin{aligned}
E^{0} & =\operatorname{Tr} \frac{1}{2 \pi i} \oint d z \frac{z}{z-H^{0}} \\
& =\operatorname{Tr} \frac{1}{2 \pi i} \oint d z \frac{|i\rangle z\langle i|}{z-\epsilon_{i}} \\
& =\sum_{i}^{o c c} \epsilon_{i} .
\end{aligned}
$$

Here, the integration contour, called the Coulson contour, ${ }^{1}$ enclosing poles corresponding to the occupied levels, or in recent many body theory, enclosing the upper half plane which includes only the hole levels with positive infinitesimal imaginary parts. ${ }^{6}$ The Einstein convention that the repeated indices imply summation is adopted. If the single-particle energy $\epsilon_{\mathrm{i}}$ is obtained by the HF (Hartree-Fock) approximation, the electron-electron interaction is doubly counted in the summation with respect to $i$, which however does not matter for a theoretical investigation, since this extra quantity merely changes the standard for the energy estimation. We also disregard the spin consideration for simplicity.

We now try the perturbation expansion for $G(z)$, 


$$
\begin{aligned}
G(z) & =\frac{1}{z-H}=\frac{1}{z-H^{0}-v} \\
& =\frac{1}{z-H^{0}}+\frac{1}{z-H^{0}} v \frac{1}{z-H} \\
& =\frac{1}{z-H^{0}}+\frac{1}{z-H^{0}} v \frac{1}{z-H^{0}} \\
& +\frac{1}{z-H^{0}} v \frac{1}{z-H^{0}} v \frac{1}{z-H^{0}}+\ldots .
\end{aligned}
$$

The relation for the second line is exact, called the Dyson equation, and the third line is the perturbation expansion. We thus have the total energy of

$$
\begin{aligned}
E= & \operatorname{Tr} \frac{1}{2 \pi i} \oint d z \frac{z}{z-H} \\
= & \operatorname{Tr} \frac{1}{2 \pi i} \oint d z\left\{\frac{z}{z-H^{0}}+\frac{1}{z-H^{0}} v \frac{1}{z-H^{0}}\right. \\
& \left.+\frac{1}{z-H^{0}} v \frac{1}{z-H^{0}} v \frac{1}{z-H^{0}}+\ldots\right\}
\end{aligned}
$$

This expression is slightly simplified, if we observe, for example,

$$
\begin{aligned}
\operatorname{Tr} \oint d z z \frac{1}{z-H^{0}} v \frac{1}{z-H^{0}} & =-\operatorname{Tr}\left[z \frac{v}{z-H^{0}}\right]_{0}^{0} \\
& +\operatorname{Tr} \oint d z \frac{v}{z-H^{0}}
\end{aligned}
$$

Substituting this into (1.7) and integrating by parts lead to

$$
\begin{aligned}
\Delta E & =\operatorname{Tr} \frac{1}{2 \pi i} \oint d z\left[\frac{z}{z-H}-\frac{z}{z-H^{0}}\right] \\
& =\operatorname{Tr} \frac{1}{2 \pi i} \oint d z\left\{v \frac{z}{z-H^{0}}+\frac{1}{2} v \frac{z}{z-H^{0}} v \frac{z}{z-H^{0}}+\ldots\right\} \\
& =\operatorname{Tr} \frac{1}{2 \pi i} \oint d z\left\{v G^{0}+\frac{1}{2} v G^{0} v G^{0}+\ldots+\frac{1}{n}\left(v G^{0}\right)^{n}+\ldots\right\} \\
& =\operatorname{Tr} \frac{-1}{2 \pi i} \oint d z \log \left(1-v G^{0}\right) \\
& =\frac{-1}{2 \pi i} \oint d z \log \operatorname{det}\left(1-v G^{0}\right) .
\end{aligned}
$$

In this,

$$
-\log \left(1-v G^{0}\right)=-\log \frac{G^{0}}{\left(G^{0}\right)^{-1}-v}=\log \frac{G}{G^{0}},
$$

where the last term is the difference between zeros and poles, and indicates the summation of infinite series implies the exact solution. However, this elegant result is almost useless.

The extra energy $\Delta E$ due to the perturbation is evaluated by using the third line of (1.9), up to the second order with respect to $v$ which is independent of $z$. Note that $G^{0}$ is usually labeled by level indices, while $v$ is labeled by site indices in the quantum chemistry. Let us begin with the first-order correction:

$$
\begin{aligned}
\Delta E^{(1)} & =\operatorname{Tr} \frac{1}{2 \pi i} \oint d z v G^{0} \\
& =\frac{1}{2 \pi i} \oint d z\langle a|v| b\rangle \frac{\langle b \mid i\rangle\langle i \mid a\rangle}{z-\epsilon_{i}} \\
& =\langle a|v| b\rangle q_{b a}=\operatorname{Tr} v q,
\end{aligned}
$$

where $q_{b a}$ is the bond order between sites $b$ and $a$, defined by

$$
\begin{aligned}
& \left\{q_{b a}\right\}=\left\{\sum_{i}^{o c c}\langle b \mid i\rangle\langle i \mid a\rangle\right\} \\
& =\sum_{i}^{o c c}\left(\begin{array}{c}
C_{1 i} \\
C_{2 i} \\
\ldots \\
C_{n i}
\end{array}\right)\left(C_{i 1} C_{i 2} \ldots C_{i n}\right) \\
& =\sum_{i}^{o c c}\left(\begin{array}{cccc}
C_{1 i} C_{i 1} & C_{1 i} C_{i 2} & \ldots & C_{1 i} C_{i n} \\
C_{2 i} C_{i 1} & C_{2 i} C_{i 2} & \ldots & C_{2 i} C_{i n} \\
C_{3 i} C_{i 1} & C_{3 i} C_{i 2} & \ldots & \\
\ldots & & &
\end{array}\right)
\end{aligned}
$$

In the last term, the LCAO coefficients in ordinary quantum chemistry are introduced, and the matrix character of the bond order is clearly given. Note that the charge density is the diagonal element of this matrix. Hereafter we call the bond order including the so called bond order and charge density. We now have the first order correction of the stabilization energy in terms of the bond orders and the interaction terms.

The second-order term is evaluated in a similar way as

$$
\begin{aligned}
\Delta E^{(2)} & =\operatorname{Tr} \frac{1}{2} \frac{1}{2 \pi i} \oint d z v G^{0} v G^{0} \\
& =\frac{1}{2} \frac{1}{2 \pi i} \oint d z\langle a|v| b\rangle \frac{\langle b \mid i\rangle\langle i \mid c\rangle}{z-\epsilon_{i}}\langle c|v| d\rangle \frac{\langle d \mid i\rangle\langle j \mid a\rangle}{z-\epsilon_{j}} \\
& \equiv v_{a b} \pi_{a b ; c d} v_{c d} .
\end{aligned}
$$

In the last line, $\pi_{a b ; c d}$ is the bond-bond polarizability, ${ }^{1}$ which is the most general among various polarizabilities. This is understood as a kind of correlation function telling how the interactions $v_{a b}$ and $v_{c d}$ coherently effect to the energy. In the present expression,

$$
\begin{aligned}
\pi_{a b ; c d} & =\frac{1}{2} \frac{1}{2 \pi i} \oint d z G_{b c}^{0}(z) G_{b a}^{0}(z) \\
& =\frac{1}{2} \frac{1}{2 \pi i} \oint d z \frac{\langle b \mid i\rangle\langle i \mid c\rangle}{z-\epsilon_{i}} \frac{\langle d \mid j\rangle\langle j \mid a\rangle}{z-\epsilon_{j}} \\
& =\sum_{i}^{\rho c c} \sum_{j} \frac{\langle b \mid i\rangle\langle i \mid c\rangle\langle d \mid j\rangle\langle j \mid a\rangle}{\epsilon_{i}-\epsilon_{j}} .
\end{aligned}
$$

The polarizability is a super matrix, each element of which is composed of a matrix; it is almost impossible to print out all of them. ${ }^{8}$ We would like to emphasize the present simple derivation, compared with the ordinal perturbation theory explicitly using the wave functions.

Numerous illustrative examples can be found in the literature. ${ }^{9}$ Here, we give a simple example. Imagine a ring polyene having ten members. If a bond resulting in naphthalene is formed, naphthalene is stable due to the first-order effect using the bond order, while in the case of azulene, this bond forming has no effect in the first order, but azulene gets a little stabilization through the second-order effect which is written in terms of the polarizability.

\section{Inter-Molecular Interaction}

The method developed in the previous chapter is now ap- 
plied to the intermolecular interaction; however in the present case the intermolecular interaction implies the ordinary chemical bonds constructed between molecules, and does not imply the dipole-dipole interaction between molecules.

The treatment of molecular interaction is carried out in two steps, depending upon how to select the non-interacting systems. Now, two molecules, $A$ and $B$, approach each other. When a bond is formed, two molecules are no longer regarded as independent system, but the combined system is regarded as a new $H^{0}$ system. The eigenvalue problem for this new system is solved. The resulting bond order matrix suggests the secondly arising bond in such a way that a preferable combination of the bond order and the coupling term arises to yield stabilization; at the same time the geometry of the molecular compound is determined. This approach has been tried by T. Kano et al. ${ }^{11}$ However this method requires a new $H^{0}$ problem. At present we present another way to avoid this complexity, in analogy with the method of orbital interaction. ${ }^{2,4,5}$

If we look at (1.8), even if only one bond is formed between $A$ and $B$, as is seen in biphenyl, the second order-procedure is required due to the Tr operation:

$$
\begin{aligned}
\Delta E_{A B}^{(2)}= & \operatorname{Tr} \frac{1}{2} \frac{1}{2 \pi i} \oint d z v G^{0} v G^{0} \\
= & \frac{1}{2} \frac{1}{2 \pi i} \oint d z\left\langle A_{1}|v| B_{1}\right\rangle \frac{\left\langle B_{1} \mid B_{i}\right\rangle\left\langle B_{i} \mid B_{1}\right\rangle}{z-\epsilon_{B_{i}}} \\
& \left\langle B_{1}|v| A_{1}\right\rangle \frac{\left\langle A_{1} \mid A_{j}\right\rangle\left\langle j_{A} \mid A_{1}\right\rangle}{z-\epsilon_{A_{j}}} \\
= & \frac{1}{2} \sum_{\left(A_{j}\right)}^{o c c} \sum_{\left(B_{i}\right)}^{u n} \frac{\left.\left\langle A_{1} \mid A_{j}\right\rangle v_{A_{1}, B_{1}}\left\langle B_{1} \mid B_{i}\right\rangle\right|^{2}}{\epsilon_{A_{j}}-\epsilon_{B_{i}}} \\
+ & \left(\text { the term with } A_{j} \leftrightarrow B_{i}\right) .
\end{aligned}
$$

This is a familiar result from the second-order perturbation theory. One may argue that this is an interaction between the partial charges, $\left|<A_{1}\right| A_{\mathrm{j}}>\left.\right|^{2}$ and $\left|<B_{1}\right| B_{\mathrm{i}}>\left.\right|^{2}$. However, we must notice that combining two couplings and two propagators makes a circle that starts from A and terminates at A. Stabilization occurs mainly from mixture of the wave functions of A and B through couplings. A solid line representing a local chemical bond between two molecules gives little informations.

We next encounter a more general case where two bonds are formed between two molecules. If the two bonds are constructed at a distance, two molecules rigidly combine with each other. The calculation is entirely the same as that previously done:

$$
\begin{aligned}
\Delta E_{A B}^{(2)}= & \operatorname{Tr} \frac{1}{2} \frac{1}{2 \pi i} \oint d z v G^{0} v G^{0} \\
= & \frac{1}{2} \frac{1}{2 \pi i} \oint d z\left(v_{A_{1}, B_{1}}\right) G_{B_{1}, B_{n}}^{0}\left(v_{B_{n}, A_{k}}\right) G_{A_{k}, A_{1}}^{0} \\
= & \frac{1}{2} \frac{1}{2 \pi i} \oint d z\left\langle A_{1}|v| B_{1}\right\rangle \frac{\left\langle B_{1} \mid B_{i}\right\rangle\left\langle B_{i} \mid B_{n}\right\rangle}{z-\epsilon_{B_{i}}} \\
& \left\langle B_{n}|v| A_{k}\right\rangle \frac{\left\langle A_{k} \mid A_{j}\right\rangle\left\langle A_{j} \mid A_{1}\right\rangle}{z-\epsilon_{A_{j}}} \\
= & \frac{1}{2} \sum_{\left(A_{j}\right)}^{o c c} \sum_{\left(B_{i}\right)}^{\text {un }} \frac{\left\langle A_{1}|v| B_{1}\right\rangle\left\langle B_{1} \mid B_{i}\right\rangle\left\langle A_{1} \mid A_{j}\right\rangle\left\langle B_{n}|v| A_{k}\right\rangle\left\langle A_{k} \mid A_{j}\right\rangle\left\langle A_{j} \mid A_{1}\right\rangle}{\epsilon_{A_{j}}-\epsilon_{B_{i}}}
\end{aligned}
$$

$$
+ \text { (the term with } A_{j} \leftrightarrow B_{i} \text { ). }
$$

Although the last result is rather complicated, the second line of (2.2) is appealing because of a clear-cut representation of the interaction scheme. A rectangle can be drawn with vertices, $A_{1}, B_{1}, B_{n}$ and $A_{k}$. The lines $\overline{A_{1} B_{1}}$ and $\overline{B_{n} A_{k}}$ are couplings, while lines $\overline{A_{1} A_{k}}$ and $\overline{B_{1} B_{n}}$ represent propagators. By imitating the result of (1.13), we may define a sort of polarizabilty,

$$
\Delta E_{A B}^{(2)}=\left(v_{A_{1}, B_{1}}\right) \pi_{A_{1}, B_{1} ; B_{n}, A_{k}}\left(v_{B_{n}, A_{k}}\right)
$$

with

$$
\begin{aligned}
\pi_{A_{1}, B_{1} ; B_{n}, A_{k}} & =\frac{1}{2} \frac{1}{2 \pi i} \oint d z G_{B_{1}, B_{n}}^{0}(z) G_{A_{k}, A_{1}}^{0}(z) \\
& =\frac{1}{2} \sum_{\left(B_{i}\right)}^{o c c} \sum_{\left(A_{j}\right)}^{o c c} \frac{\left\langle B_{1} \mid B_{i}\right\rangle\left\langle B_{i} \mid B_{n}\right\rangle\left\langle A_{k} \mid A_{j}\right\rangle\left\langle A_{j} \mid A_{1}\right\rangle}{\epsilon_{A_{j}}-\epsilon_{B_{i}}} \\
& +\left(\text { the term with } A_{j} \leftrightarrow B_{i}\right) .
\end{aligned}
$$

The similar discussion as that after (2.1) is presented. It must be stressed that (2.4) is constructed only from the terms $H_{A}^{0}$ and $H_{B}^{0}$.

\section{Effective Interaction}

The Dyson equation for Green's function is

$$
\begin{aligned}
G & =G^{0}+G^{0} v G \\
& =G^{0}+G^{0} v G^{0}+G^{0} v G^{0} v G^{0}+G^{0} v G^{0} v G^{0} v G^{0}+\ldots .
\end{aligned}
$$

We now rewrite this relation as

$$
G=G^{0}+G^{0} \tilde{v} G^{0}
$$

defining an effective interaction $\tilde{v}$. At a glance we recognize

$$
\tilde{v}=v+v G^{0} v+v G^{0} v G^{0} v+\ldots=v+v G^{0} \tilde{v}
$$

This is solved formally as

$$
\tilde{v}(z)=\frac{v}{1-v G^{0}(z)} .
$$

However, from the viewpoint of chemical reactivity, the perturbative expansion is preferable for us,

$$
\begin{aligned}
\tilde{v}_{A_{k}, A_{l}}(z) & \approx v_{A_{k}, A_{l}}+v_{A_{k}, B_{m}} G_{B_{m}, B_{n}}^{0} v_{B_{n}, A l} \\
& =v_{A_{k}, A_{l}}+\frac{\left\langle A_{k}|v| B_{m}\right\rangle\left\langle B_{m} \mid B_{J}\right\rangle\left\langle B_{j} \mid B_{n}\right\rangle\left\langle B_{n}|v| A_{l}\right\rangle}{z-\epsilon_{B_{j}}} .
\end{aligned}
$$

If we multiply this by $G_{A_{l}, A_{k}}^{0}(z)$ and integrate over $z$, the result (2.1) is restored.

However, a conventional analysis is possible by decoupling the above procedures. If we are now interested in the ground state of $A$, the main effects for stabilization arise from replacements,

$$
z \sim \epsilon_{A_{H}}, \quad \epsilon_{j_{B}} \sim \epsilon_{B_{L}},
$$


where $\epsilon_{A_{H}}$ is the energy of the highest occupied level of $A$ and $\epsilon_{B_{L}}$ is that of the lowest vacant level of $B$. Then, the original interaction $v_{A_{k}, A_{l}}$ is modified by an effect due to the additional intermolecular interaction, as

$$
\begin{aligned}
\Delta v_{A_{k}, A_{l}} & \approx \frac{\left\langle A_{k}|v| B_{m}\right\rangle\left\langle B_{m} \mid B_{L}\right\rangle\left\langle B_{L} \mid B_{n}\right\rangle\left\langle B_{n}|v| A_{l}\right\rangle}{\epsilon_{A_{H}}-\epsilon_{B_{L}}} \\
& =v_{A_{k}, B_{m}} \tilde{q}_{B_{m}, B_{n}} v_{B_{n}, A_{l}} .
\end{aligned}
$$

Here, by the way, we introduced the effective bond order in view of the correlation feature,

$$
\tilde{q}_{B_{m}, B_{n}}=\frac{\left\langle B_{m} \mid B_{L}\right\rangle\left\langle B_{L} \mid B_{n}\right\rangle}{\epsilon_{A_{H}}-\epsilon_{B_{L}}} .
$$

However, this would physically refer to the polarizability.

We then multiply (3.5) by the bond order $q_{A}, A_{k}$, giving the stabilization energy,

$$
\Delta E=q_{A_{l}, A_{k}} \Delta v_{A_{k}, A_{l}} .
$$

Let us investigate a simple example, a molecular compound between ethylene and $\mathrm{H}_{2}$, which are denoted $A$ and $B$, respectively (see Fig. 1). First, we note

$$
q_{A_{1}, A_{2}}=\frac{1}{2}>0 \text {. }
$$

In this case, (3.5) is written as

$$
\tilde{v}_{A_{2}, A_{1}}=v_{A_{2}, B_{2}} \frac{\left\langle B_{2} \mid B_{L}\right\rangle\left\langle B_{L} \mid B_{1}\right\rangle}{\epsilon_{A_{H}}-\epsilon_{B_{L}}} v_{B_{1}, A_{1}} .
$$

It is probable that $\epsilon_{A_{H}-} \epsilon_{B_{L}}<0$, and $<B_{2} \mid B_{L}>$ and $<B_{L} \mid B_{1}>$ are out of phase, say $<B_{2}\left|B_{L}><B_{L}\right| B_{1}><0$. If we consider aboves, the condition that $\Delta E$ be negative or the effective interaction $\Delta v_{A_{2}, A_{1}}$ be negative requires $v_{A_{2}, B_{2}} v_{A_{1}, B_{1}}<0$. Namely, these are different in sign. Graphically speaking, as expressed by Fig. 1, an atom of $\mathrm{H}_{2}$ approaches from the upper side of the ethyle surface, and the other from the lower side. The precise calculation has been made by Motoki. ${ }^{10}$ It is amusing to point out that this might be the smallest Möius band.

In a real problem, a synthetic technique to make a specified

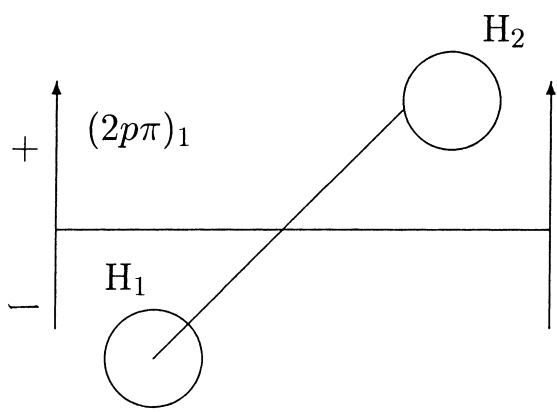

$(2 p \pi)_{2}$

Fig. 1. Molecular compound between ethylene and the $\mathrm{H}_{2}$ molecule.

The arrows represent the $2 \mathrm{p}_{\pi}$ AOs of carbon atoms, while the circles the $1 \mathrm{~s}$ AOs of the hydrogen molecule. This is perhaps the smallest Möius band. bond stronger or weaker, or sometimes to break it, is wanted. From the viewpoint of an effective interaction, this might be possible, to some extent, by introducing another perturbation, $<A_{s}|v| A_{t}>$, which effects the bond order, $q_{A_{l} A_{k}}$. To this end, let us return to (1.6) and take the first-order effect into account. The correction of the bond order is given as (for a while we omit index $A$, limiting considerations inside $A$.)

$$
\begin{aligned}
\Delta q_{k 1} & =\frac{1}{2 \pi i} \oint d z\left(G_{k 1}(z)-G_{k 1}^{0}(z)\right) \\
& =\frac{1}{2 \pi i} \oint d z\left\langle k\left|\frac{1}{z-H^{0}} v \frac{1}{z-H^{0}}\right| 1\right\rangle \\
& =\frac{1}{2 \pi i} \oint d z \frac{\langle k \mid i\rangle\langle i \mid r\rangle}{z-\epsilon_{i}}\langle r|v| s\rangle \frac{\langle s \mid j\rangle\langle j \mid 1\rangle}{z-\epsilon_{j}} \\
& =2 \sum_{i}^{o c c u n} \frac{\langle k \mid i\rangle\langle i \mid r\rangle\langle r|v| s\rangle\langle s \mid j\rangle\langle j \mid 1\rangle}{\epsilon_{i}-\epsilon_{j}} \\
& =v_{r s} \pi_{r s ; k 1} .
\end{aligned}
$$

\section{Photo-Chemistry}

In this chapter we discuss the chemical reactivity in the radiation field. The Hamiltonians responsible for this problem are

$$
\begin{aligned}
& H_{0}=\epsilon_{i} a_{i}^{+} a_{i}+\omega_{k} c_{k}^{+} c_{k}, \\
& H^{i n t}=f_{i j ; k} a_{i}^{+} a_{j}\left(c_{k}^{+}+c_{k}\right),
\end{aligned}
$$

where the usual notations are used. According to the discussion in the previous chapters, we aim to obtain the bond order of the molecule in question in the radiation field. To facilitate manipulation, we introduce projection operators,

$$
P=\sum_{i}^{o c c}|i\rangle\left\langle i\left|. \quad Q=\sum_{l}^{u n}\right| l\right\rangle\langle l| .
$$

It is obvious that

$$
\begin{aligned}
& P+Q=1, \\
& P^{2}=P, \quad Q^{2}=Q, \\
& P Q=Q P=0 .
\end{aligned}
$$

The propagator $G$ is written as

$$
G=(P+Q) G(P+Q)=G^{P}+G^{Q}+G^{P Q}+G^{Q P} .
$$

We deal with these elements in turn. When we project out the perturbation expansion of the propagator,

$$
G=G^{0}+G^{0} H^{i n t} G^{0}+G^{0} H^{i n t} G^{0} H^{i n t} G^{0}+\ldots,
$$

by $P$ for example, since the non-interacting propagator $G^{0}$ is diagonal to $P$ and $Q$, or $P G^{0} Q=0$, the term of the lowest order to $H^{\text {int }}$ begins with the second-order term of $H^{\text {int }}$. We then have the Dyson equation, such as

$$
G^{P}(z)=G_{0}^{P}(z)+G_{0}^{P}(z)\left(H^{i n t}\right)^{P Q} G_{0}^{\mathrm{Q}}(z)\left(H^{i n t}\right)^{Q P} G^{P}(z) .
$$

This is solved as

$$
G^{P}(z)=\left[\left(G_{0}^{P}(z)\right)^{-1}-\left(H^{i n t}\right)^{P Q} G_{0}{ }^{Q}(z)\left(H^{i n t}\right)^{Q P}\right]^{-1} .
$$


Similarly, we have $G^{Q}(z)$,

$$
G^{Q}(z)=\left[\left(G_{0}^{Q}(z)\right)^{-1}-\left(H^{i n t}\right)^{Q P} G_{0}^{P}(z)\left(H^{i n t}\right)^{P Q}\right]^{-1} .
$$

On the other hand, $G^{P Q}$ is slightly more complicated. The Dyson equation for this is

$$
G^{P Q}=G_{0}^{P}\left(H^{i n t}\right)^{P Q} G_{0}^{Q}+G^{P}\left(H^{i n t}\right)^{P Q} G^{Q}\left(H^{i n t}\right)^{Q P} G^{P Q},
$$

from which

$$
G^{P Q}=\left[\left(G_{0}^{P}\left(H^{i n t}\right)^{P Q} G_{0}^{Q}\right)^{-1}-H^{i n t}\right]^{-1} .
$$

Therefore, because $G^{P Q}$ and $G^{Q P}$ have small effects on the total $G$ compared with $G^{P}$ and $G^{Q}$, they are neglected in the following.

The second term on the right-hand side of (4.6) is called the self-energy part of $G^{P}$,

$$
M^{P}(z)=\left(H^{i n t}\right)^{P Q} G_{0}^{Q}(z)\left(H^{i n t}\right)^{Q P} .
$$

We investigate this in detail. The interaction matrices are as follows:

$$
\begin{aligned}
& \left(H^{i n t}\right)^{Q P}:\left\langle l, n_{k}-1\left|H^{i n t}\right| i, n_{k}\right\rangle=f_{l i, k} \sqrt{n_{k}}, \\
& \left(H^{i n t}\right)^{P Q}:\left\langle i, n_{k}\left|H^{i n t}\right| l, n_{k}-1\right\rangle=f_{i l, n_{k}} \sqrt{n_{k}},
\end{aligned}
$$

where the hole (occupied) and particle (unoccupied) levels are represented by indices $i$ and $l$, respectively, and for $\left(H^{i n t}\right)^{Q P}$ the absorption process is employed, while for $\left(H^{\text {int }}\right)^{P Q}$ the emission process is employed. Here, we used the relations,

$$
c_{k}\left|n_{k}\right\rangle=\sqrt{n_{k}}\left|n_{k}-1\right\rangle, c_{k}^{+}\left|n_{k}-1\right\rangle=\sqrt{n_{k}}\left|n_{k}\right\rangle
$$

We then have

$$
M^{P}(z)=\frac{n_{k}\left|f_{l i, k}\right|^{2}}{z-\epsilon_{l}-\left(n_{k}-1\right) \omega_{k}} .
$$

In the above, because the inside propagator refers to $Q$ (particle state), $\epsilon_{l}$ should be $\epsilon_{l}-i \eta$, where $\eta$ a positive infinitesimal. ${ }^{6,7}$ Precisely speaking, the particle has the boundary condition that it decays in the infinite future (at $t=+\infty$ ),

$$
e^{-i\left(\epsilon_{1}-i \eta\right) t}=e^{-i \epsilon_{l} t} e^{-\eta t} \rightarrow 0(t=+\infty) .
$$

On the other hand, the hole decays at $t=-\infty$, or $\epsilon_{i}+i \eta$. Note that the level energies are all set to be positive. The particle propagates to the future, while the hole propagates backward in time. Further an approximation is made: because we are now interested in the hole states denoted by $P, z$ in (4.12) may be replaced by $\epsilon_{i}+n_{k} w_{k}$, and the imaginary part is displayed as

$$
\epsilon_{i}+i \eta-\left(\epsilon_{l}-i \eta\right)=\epsilon_{i l}+i \eta, \quad \epsilon_{i l}=\epsilon_{i}-\epsilon_{l} .
$$

We thus have

$$
M^{P}(z)=\frac{n_{k}\left|f_{l i, k}\right|^{2}}{\epsilon_{i l}+\omega_{k}+i \eta} .
$$

At this stage, we consider the sum over $\mathbf{k}$, or integration over $\omega_{k}$ with a suitable state density $\rho\left(\omega_{k}\right)$, leading to

$$
\begin{aligned}
M^{P}(z) & =n_{k}\left|f_{l i, k}\right|^{2}\left\{P \frac{1}{\epsilon_{i l}+\omega_{k}}-i \pi \delta\left(\epsilon_{i l}+\omega_{k}\right)\right\} \\
& \approx-i \pi \delta\left(\epsilon_{i l}+\omega_{k}\right) n_{k}\left|f_{l i, k}\right|^{2},
\end{aligned}
$$

where the principal part integration is neglected, since it causes only a small level shift. ${ }^{7}$ Note that $M^{P}$ gives the negative imaginary part; if we take this into account in (4.6), it is found that the hole level gets the negative imaginary part, $i \pi \delta\left(\epsilon_{i l}+\right.$ $\left.\omega_{k}\right) n_{k}\left|f_{l i, k}\right|^{2}$, which is physical. In other words, due to the radiation effect, the hole level $i$ is lifted to an excited level $l$.

In a similar way we obtain

$$
\begin{aligned}
M^{Q}(z) & =n_{k}\left|f_{l i, k}\right|^{2} \frac{1}{z-\epsilon_{i}-n_{k} \omega_{k}} \\
& =n_{k}\left|f_{l i, k}\right|^{2} \frac{1}{\epsilon_{l i}-\omega_{k}-i \eta} \\
& =n_{k}\left|f_{l i, k}\right|^{2}\left\{P \frac{1}{\epsilon_{l i}-\omega_{k}}+i \pi \delta\left(\epsilon_{l i}-\omega_{k}\right)\right\} \\
& \approx+i \pi \delta\left(\epsilon_{l i}-\omega_{k}\right) n_{k}\left|f_{l i, k}\right|^{2} .
\end{aligned}
$$

Note that $M^{Q}$ gets the positive imaginary part instead of the negative one in $M^{P}$.

Bond Order. We are well equipped to evaluate the bond order in the radiation field. The bond order $q_{r s}$ is obtained as the matrix element of $G(z)$,

$$
\begin{aligned}
q_{r s} & =\frac{1}{2 \pi i} \oint d z\langle r|G(z)| s\rangle \\
& \approx \frac{1}{2 \pi i} \oint d z\left\langle r\left|G^{P}\right| s\right\rangle+\left\langle r\left|G^{Q}(z)\right| s\right\rangle,
\end{aligned}
$$

neglecting $G^{P Q}$ and $G^{Q P}$. First we evaluate

$$
\begin{aligned}
& \frac{1}{2 \pi i} \oint d z\left\langle r\left|G^{P}(z)\right| s\right\rangle \\
& =\frac{1}{2 \pi i} \oint d z\left\langle r \mid i, n_{k}\right\rangle\left(\frac{1}{z-\epsilon_{i}-i \eta+i \pi \delta\left(\epsilon_{i^{\prime} l^{\prime}}+\omega_{k}\right)}\right)\left\langle i, n_{k} \mid s\right\rangle .
\end{aligned}
$$

Here, we observe an interesting result that in the absence of radiation, every hole level has the positive imaginary part, but in the radiation field, one of these satisfying $\delta\left(\epsilon_{i^{\prime} l^{\prime}}+\omega_{k}\right)$ has the negative imaginary part. We hereafter call $i^{\prime}$ and $l^{\prime}$ as $H$ and $L$, respectively. Upon integraton, we enclose the contour on the upper half half plane of $z$, which is forced by the hidden convergence factor, $\exp \left(-i z 0^{-}, z \rightarrow i \infty\right)$.

$$
\frac{1}{2 \pi i} \oint d z\left\langle r\left|G^{P}(z)\right| s\right\rangle=q_{r s}-q_{r s}^{H},
$$

where $q_{r s}^{H}$ is the partial bond order with respect to $H$.

Similarly,

$$
\frac{1}{2 \pi i} \oint d z\left\langle r\left|G^{Q}(z)\right| s\right\rangle=q_{r s}^{L} .
$$


We thus have the bond order in the radiation field,

$$
\begin{aligned}
\frac{1}{2 \pi i} \oint d z(\langle r|G(z)| s\rangle & =\frac{1}{2 \pi i} \oint d z\left(\left\langle r\left|G^{P}(z)\right| s\right\rangle+\left\langle r\left|G^{Q}(z)\right| s\right\rangle\right) \\
& =q_{r s}+\left(q_{r s}^{L}-q_{r s}^{H}\right) .
\end{aligned}
$$

The second term of the above expression is an additional bond order due to the the radiation field. We consider that the chemical reactivity in the radiation is determined by this additional factor,

$$
q_{r s}^{L H}=q_{r s}^{L}-q_{r s}^{H} .
$$

Fukui $^{2}$ has discussed the chemical reactivity in the radiation field only in terms of $q_{r s}^{L}$; however, we would rather consider that $L$ and $H$ contribute with equal weights to the result. Our result shows that this is just the case, but with opposite phases. In the case of alternant hydrocarbons, for the active sites, $q_{r s}^{H}=$ $-q_{r s}^{L}$. Therefore, the present result coincides with Fukui's assertion.

In discussing the chemical reactivity for the ground state (thermo-chemistry), we developed a theory that is invariant under the cootdinate transformation in the Hilbert space; however, in the present case, the result depends upon the specified orbitals, $H$ and $L$. We now consider the effect of the radiation field: the polarization of light makes the shapes of orbitals canonical, and the resonace frequency $\omega_{k}$ selects $H$ and $L$ according to the condition $\delta\left(\epsilon_{L}-\epsilon_{H}-\omega_{k}\right)$, so that $H$ and $L$ are now physical.

\section{Applications}

The present theory is based on the energy consideration developed under the Tr operation. As is well known, Tr operation ensures that the result is invariant under any transformation of orbitals as long as all orbitals take part in. However, the theory in the radiation field works with specified orbitals connected by the radiation field. This problematic point in view of the previous assertion, however, is properly accepted, since in the radiation field the so-called canonical orbitals become physically meaningful.

We now consider the molecular interaction between $A$ and $B$ molecules. The quantum mechanical properties of these molecules are determined by themselves, independently. For both molecules the eigen-values and eigen-functions are prepared independently. We then set some couplings between them, and carry out calculations pertubatively, or as an eigen-value problem. Then if a preferable result to stabilize the whole system is obtained, we conclude that the reaction in question is likely to take place. However, this speculation concerning the reactivity theory is considerably unsatisfactory. Look at (2.3) or (3.5). For example, the first one indicates that the extra energy consists of two parts, the polarizabilty and couplings. The polarizability is written by using unperturbed terms of the respective molecules. Thus if we wish to obtain a new molecule as a stable substance, the qualitative natures of the couplings are fatally determined. We have thus presented an unusual structure for the ethlene- $\mathrm{H}_{2}$ compound as the smallest Möius band.

An easier analysis of this compound is reviewed by using the effective interaction. In this molecule we can define an effective coupling due to the intermolecular interaction,

$$
\tilde{v}_{A 2, A 1}=v_{A_{2}, B_{2}} \frac{\left\langle B_{2} \mid B_{L}\right\rangle\left\langle B_{L} \mid B_{1}\right\rangle}{\epsilon_{A_{H}}-\epsilon_{B_{L}}} v_{B_{1}, A_{1}} .
$$

The extra energy obtained in dark (thermally) is given as

$$
\Delta E=q_{A B} \widetilde{v}_{B A} .
$$

However, in the radiation field, since the bond order acquires an additional term $q_{A_{1}, A_{2}}^{L H}$ as is seen in (4.20), the change of the extra energy $\Delta E^{f}$ amounts to

$$
\Delta E^{f}=q_{A_{2} A_{1}}^{L H} \tilde{v}_{A_{1} A_{2}} .
$$

Considering that $q_{A_{2} A_{1}}^{L H}<0$ against $q_{A_{1} A_{2}}>0$, we can conclude that the end coupling terms, $v_{A_{2} B_{2}}$ and $v_{A_{1} B_{1}}$ have the same signs.

Various applications have been made. ${ }^{11,12}$ It is claimed that the face-to-face coupling between two benzene molecules is unlikely, to displace each other by about half width of the surface; a similar displacement between two chain molecules is also theoretically obtained in accordance with observations in molecular crystals.

\section{References}

1 C. A. Coulson and H. C. Longuet-Higgins, Proc. Roy. Soc., A191, 39 (1947).

2 K. Fukui, "Chemical Reaction and Electron Orbitals," Maruzene, Tokyo, 1976 (in Japanese).

3 R. B. Woodward and R. Hoffmann, "The Conservation of Orbital Symmetry," Academic Press (1970).

4 H. Fujimoto, N. Koga, and K. Fukisi, J. Am. Chem. Soc., 103, 196 (1981).

5 T. Ohmae, K. Nishikawa, K. Deguchi, and S. Aono, Bull. Chem. Soc. Jpn., 54, 2857 (1981).

6 A. L. Fetter and J. D. Wallecka, "Quantum Theory of Many-Particle Systems," McGraw-Hill, New York (1971).

7 R. D. Mattuck, "A guide to Feynman diagrams in the many-body problem," McGraw-Hill, New York (1976).

8 C. A. Coulson et al. "Dictionary of p-Electron Calculation," Pergamon Press (1965).

9 M. Yamamoto, K. Nishikawa, and S. Aono, Bull. Chem. Soc. Jpn., 58, 3176 (1985).

10 T. Motoki, unpublished.

11 T. Kano, K. Nishikawa, and S. Aono, Bull. Chem. Soc. Jpn., 60, 2817 (1985).

12 S. Aono, Prog. Theoret. Phys., 20, 133 (1958) ; 103, 196 (1981) 